\title{
Management of increased systemic flow requirements in patients with left ventricular assist devices
}

\author{
Sascha Ott ${ }^{1}$, Pia Lanmüller ${ }^{2,3}$, Gaik Nersesian ${ }^{2,3}$, Christoph T. Starck ${ }^{2,3,4}$, Benjamin O'Brien ${ }^{1,5,6}$, \\ Volkmar Falk ${ }^{2,3,7,8,9}$, Evgenij Potapov ${ }^{2,3}$ \\ ${ }^{1}$ Department of Cardiac Anesthesiology and Intensive Care Medicine, German Heart Center Berlin, Berlin, Germany; ${ }^{2}$ Department of \\ Cardiothoracic and Vascular Surgery, German Heart Center Berlin, Berlin, Germany; ${ }^{3}$ DZHK (German Centre for Cardiovascular Research), \\ Partner Site Berlin, Berlin, Germany; ${ }^{4}$ Steinbeis University Berlin, Institute (STI) of Cardiovascular Perfusion, Berlin, Germany; ${ }^{5}$ Department of \\ Cardiac Anesthesiology and Intensive Care Medicine, Charité-Universitätsmedizin Berlin, Berlin, Germany; ${ }^{6}$ William Harvey Research Institute, \\ London, UK; ${ }^{7}$ Department of Cardiothoracic Surgery, Charité-Universitätsmedizin Berlin, Berlin, Germany; ${ }^{8}$ Berlin Institute of Health at Charité - \\ Universitätsmedizin Berlin, Berlin, Germany; ${ }^{9}$ Translational Cardiovascular Technologies, Institute of Translational Medicine, Department of Health \\ Sciences and Technology, Swiss Federal Institute of Technology (Eth), Zürich, Switzerland \\ Correspondence to: Sascha Ott, MD. Department of Cardiac Anesthesiology and Intensive Care Medicine, German Heart Center Berlin, \\ Augustenburger Platz 1, 13353 Berlin, Germany. Email: sott@dhzb.de.
}

Submitted Apr 28, 2021. Accepted for publication May 10, 2021.

doi: 10.21037/acs-2021-cfmcs-37

View this article at: http://dx.doi.org/10.21037/acs-2021-cfmcs-37

\section{Introduction}

Left ventricular assist devices (LVAD) have become a wellestablished therapy for end-stage heart failure. Usually, LVAD flows vary between 3.5-6 L/min, regardless of the pump type or initial surgical implantation technique.

In systemic inflammatory response syndrome (1), sepsis (2) or post-resuscitation status (3), the actual demand for systemic blood flow may exceed normal and usually adequate baseline cardiac output. In heart-healthy patients, these physiological challenges are compensated for by an increase in cardiac output (1). LVAD patients however, may fail to meet systemic flow demands above baseline due to relatively fixed pump flow. Elevated lactate levels or a low mixed venous oxygen saturation, as a sign of insufficient tissue oxygenation, may be helpful in evaluating these situations $(4,5)$. However, there is no established evidence base to guide the management of such scenarios. We describe possible strategies to meet such increased systemic flow demands.

\section{Escalation of an existing LVAD system}

Depending on right ventricular (RV) function, volume status, and inotropic support, the LVAD flow may be increased, within the device's design limits, by adjusting the pump speed. Additionally, a slight increase in native left ventricular (LV) output might be possible through inotropic support. However, these interventions often have limited potential to increase systemic flow and effect physiological improvement.

\section{Veno-arterial extracorporeal life support (VA-ECLS) as an LVAD upgrade}

If the body's metabolic demand exceeds the capability of the LVAD to deliver adequate systemic blood flow, a temporary combination with VA-ECLS may be beneficial to meet tissue oxygen requirements. For the ECLS setting we recommend standard heparin-coated cannulas, a standard centrifugal pump (e.g., Rotaflow, Getinge, Sweden) and a standard membrane oxygenator all for long-term use (e.g., A.L.ONE ECMO Oxygenator, Eurosets, Italy). We prefer echo-guided, percutaneous insertion if possible, however, a surgical cut-down technique is also adequate. While both systems provide blood flow to the systemic circulation, their combination is challenging. Since VA-ECLS reduces the $\mathrm{RV}$ preload, $\mathrm{LV}$ preload is reduced as well, resulting in a "steal phenomenon" to the LVAD. Additionally, the aortic outflow of the VA-ECLS causes an increased afterload to the LVAD, resulting in a "pressure phenomenon". As 
a result, these "steal/pressure phenomenon" might have deleterious effects on flow dynamics through the LVAD and could increase the risk of pump thrombosis.

Therefore, adequate RV function to provide sufficient preload to the LVAD, and well-managed volume status are critically important.

\section{Triple mechanical circulatory support (MCS) system approach in LVAD patients with increased systemic flow requirements and concomitant RV dysfunction}

RV function is often impaired in LVAD patients and might also be affected by the underlying mechanism that led to the increased cardiac output demand (6). Under VA-ECLS support RV dysfunction further decreases the LV preload and thus reduces LVAD flow. Temporary right ventricular assist devices (tRVAD), e.g., Centrimag (Abbott, Chicago, USA) are one way of ensuring sufficient LV preload and bridging to recovery of RV function. However, combining a tRVAD with an LVAD will not increase the LVAD flow beyond the inherent design limits for the device. To achieve a higher total aortic blood flow in cases with impaired RV function, combining tRVAD, LVAD and VA-ECLS, as a triple MCS strategy, is a possible solution.

LVAD and VA-ECLS interact with one another and complement each other as outlined above, whilst tRVAD ensures sufficient LV preload and protects LVAD flow. Additionally, in combination with VA-ECLS, tRVAD contributes to $\mathrm{RV}$ unloading, thereby supporting $\mathrm{RV}$ recovery.

However, adding a third MCS increases the complexity considerably and calls for careful and ongoing evaluation of MCS interaction.

\section{Concomitant respiratory failure}

Concomitant respiratory failure adds considerable complexity when combining LVAD and VA-ECLS. Poorly oxygenated blood from the lungs is ejected by the LVAD, leading to Harlequin syndrome (7). Tailored respiratory support in this setting, again depends on the RV function.

When the RV function is sufficient, VA-ECLS can be escalated to VAV-ECLS by adding another outflow cannula for a venous return of oxygenated blood. However, splitting ECLS return, trades at the expense of aortic blood flow.

In case of RV insufficiency and escalation to the triple
MCS approach, a second oxygenator can be added to the tRVAD. Operating two oxygenators increases the complexity of respiratory support significantly and requires a differentiated evaluation of each oxygenator's output. The oxygenated blood of the tRVAD circuit will become mixed with native RV output blood and passes through the lungs. The downstream blood gas status of the LVAD + tRVAD system can be monitored by blood gas analysis sampled from a right upper limb arterial line. The blood gas status of the VA-ECLS can be monitored by taking post-oxygenator blood gas samples. Of note, excluding the VA-ECLS oxygenator to simplify the triple MCS approach is not an option, since this would lead to an extracorporeal right-to-left shunt via the ECLS.

\section{De-escalation strategy}

Once the demand for total aortic blood flow decreases and/ or lung function improves, de-escalation from a dual or triple MCS approach can be initiated.

In an LVAD + VA-ECLS approach, de-escalation can be achieved by reducing the ECLS support in line with decreasing flow demand and RV function.

A triple MCS approach creates opportunities for subsequent de-escalation strategies, especially in patients with impaired RV function. Weaning of VA-ECLS can be undertaken with regard to the systemic hemodynamics. The ensuing additional burden on the RV caused by increasing $\mathrm{RV}$ preload can be managed by adjusting and possibly upregulating the tRVAD as necessary. This allows for faster VA-ECLS weaning and, consequently, simplification of the MCS set-up. Further stepwise de-escalation of oxygenator support and tRVAD weaning can follow local tRVAD weaning protocols.

\section{Conclusions}

Where a patient's actual demand for total systemic blood flow exceeds the capability of an LVAD, a combination strategy with VA-ECLS and tRVAD can be helpful. Combining multiple circulatory support devices is complex and carries significant risk. This is particularly true of the triple MCS approach described here, which based on current experience and data should only be considered in cases where all other established therapeutic options have been exhausted and no ethical or medical ceilings of care have been identified. 


\section{Acknowledgments}

Funding: None.

\section{Footnote}

Conflicts of Interest: SO declares Research and study funds from Novartis Pharma GmbH. CTS declares payment to his institution related to his activity as speaker fees, honoraria, consultancy, advisory board fees, investigator, committee member from Angiodynamics, Abiomed, Medtronic, Philips (Spectranetics), Biotronik, Liva Nova (Sorin) and Cook Medical and departmental or institutional research funding from Cook Medical. BO Research funding from the British Heart Foundation and the National Institute for Health Science Research. Consultant for Teleflex. VF declares relevant financial activities outside the submitted work with following commercial entities: Medtronic GmbH; Biotronik SE \& Co.; Abbott GmbH \& Co. KG; Boston Scientific; Edwards Lifesciences; Berlin Heart; Novartis Pharma GmbH; JOTEC GmbH; Zurich Heart. In relation to: Educational Grants (including travel support); Fees for lectures and speeches; Fees for professional consultation; Research and study funds. EP serves as proctor and consultant for Abbott, Medtronic and Abiomed and received institutional grants from Abbott, Medtronic, Abiomed. The other authors have no conflicts of interest to declare.

Open Access Statement: This is an Open Access article distributed in accordance with the Creative Commons Attribution-NonCommercial-NoDerivs 4.0 International License (CC BY-NC-ND 4.0), which permits the non- commercial replication and distribution of the article with the strict proviso that no changes or edits are made and the original work is properly cited (including links to both the formal publication through the relevant DOI and the license). See: https://creativecommons.org/licenses/by-nc-nd/4.0/.

\section{References}

1. Stokes CD, Blevins S, Siegel JH, et al. Prediction of arterial blood gases by transcutaneous $\mathrm{O} 2$ and $\mathrm{CO} 2$ in critically ill hyperdynamic trauma patients. J Trauma 1987;27:1240-60.

2. Gahhos FN, Chiu RC, Bethune D, et al. Hemodynamic responses to sepsis: hypodynamic versus hyperdynamic states. J Surg Res 1981;31:475-81.

3. Menegazzi JJ, Ramos R, Wang HE, et al. Postresuscitation hemodynamics and relationship to the duration of ventricular fibrillation. Resuscitation 2008;78:355-8.

4. Janotka M, Ostadal P. Biochemical markers for clinical monitoring of tissue perfusion. Mol Cell Biochem 2021;476:1313-26.

5. Pinsky MR. Targets for resuscitation from shock. Minerva Anestesiol 2003;69:237-44.

6. Løgstrup BB, Nemec P, Schoenrath F, et al. Heart failure etiology and risk of right heart failure in adult left ventricular assist device support: the European Registry for Patients with Mechanical Circulatory Support (EUROMACS). Scand Cardiovasc J 2020;54:306-14.

7. Abrams D, Combes A, Brodie D. Extracorporeal membrane oxygenation in cardiopulmonary disease in adults. J Am Coll Cardiol 2014;63:2769-78.
Cite this article as: Ott S, Lanmüller P, Nersesian G, Starck CT, O'Brien B, Falk V, Potapov E. Management of increased systemic flow requirements in patients with left ventricular assist devices. Ann Cardiothorac Surg 2021;10(3):399-401. doi: 10.21037/acs-2021-cfmcs-37 